\title{
THE ACCURACY OF A DRY GAS METER TO MONITOR VENTILATION DURING EXERCISE
}

\author{
A. BONEN, B.A., M.S., Ph.D. \& A. N. BELCASTRO, B.P.E. \\ Human Performance Research Laboratory, School of Physical Education, \\ Dalhousie University, Halifax, Nova Scotia, Canada
}

\section{Introduction}

Several reports (Cunningham, 1969; Henry and Katch, 1972; Mayhew, 1973; Wilmore, 1968; Wilmore and Costill, 1974) have described procedures to measure pulmonary ventilation $\left(\dot{V}_{\mathrm{e}}\right)$ continuously during exercise from a Parkinson-Cowan-CD4 dry gas meter (PC-CD4). Since the accuracy of the calculated oxygen consumption is determined in part by the accuracy of the measured $\dot{V}_{e}$, it is critical that the PC-CD4 readings are reliable and accurate. According to manufacturer-supplied specifications the PC-CD4 meter is reliable for continuous flow rates up to $183.3 \mathrm{l} / \mathrm{min}$. During exercise, when intermittent flow rates may be as high as $150-200 \mathrm{l} / \mathrm{min}$, the maximal recommended capacity of the PC-CD4 is exceeded. Yet, it has been our experience that a PC-CD4 meter can provide reliable and accurate results. The purpose of this report is to relate some empirical evidence which has prompted us to utilize the PC-CD4 meter to monitor $\dot{V}_{\mathrm{e}}$ during exercise.

\section{Method}

A PC-CD4 meter and a 600 litre Tissot gasometer were connected in series (Figure 1). Intermittent flow rates were induced by exercising a subject on a treadmill or bicycle ergometer and permit breathing through a respiratory valve into the two gas meters (Figure 1). A large range of $\dot{V}_{e}$ was induced by altering, in a random fashion, the treadmill speed or bicycle ergometer loads from 2 to $13 \mathrm{mph}$ or from 600 to $1500 \mathrm{kpm} / \mathrm{min}$, respectively. At each workload the subjects exercised for 70 seconds. During the first ten seconds the expired volume was shunted, past the gas meters via control valve $A$. This valve was then turned to divert the expired gas volume into the gas meters. At the end of one full minute of exercise the expired gas volume was again shunted past the gas meters, inlet valve $B$ on the Tissot gasomeier was closed, and the subject stopped exercising.

$\dot{V}_{\mathrm{e}}$ 's were recorded from a potentiometric output onto a strip chart recorder and gas temperatures were monitored via a calibrated thermister located in the expiratory air stream. The resultant volumes in the Tissot gasometer were calculated from the change in height of the Tissot bell. Gas temperatures were obtained from a thermometer located in the bell.

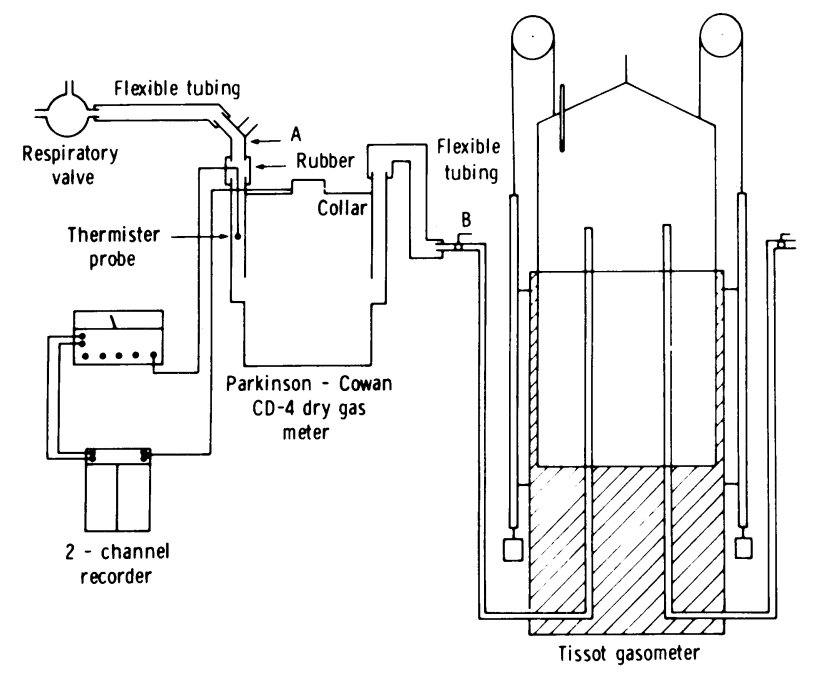

Fig. 1. Schematic representation of the system used to calibrate the Parkinson-Cowan CD-4 dry gas meter.

\section{Results}

Over the range of gas volumes $(30-193 \mathrm{l} / \mathrm{min})$, the values from the PC-CD4 were slightly but significantly lower than the Tissot values ( $<<0.05)$, the mean difference was $5.23 \pm 2.10$ litres (STPD). Comparison of the Tissot with the PC-CD4 values yielded a very high correlation coefficient $(r=0.999)$ since the correlation was forced by the experimental procedures. However, the small standard error of estimate, $\pm 1.69 \mathrm{l} / \mathrm{min}$, indicated that the PC-CD4 readings paralleled the Tissot readings closely. When a linear prediction equation was used to correct PC-CD4 values to the criterion or Tissot values (Figure 2), a very small absolute error resulted, $1.65 \pm 1.58 \%$. 


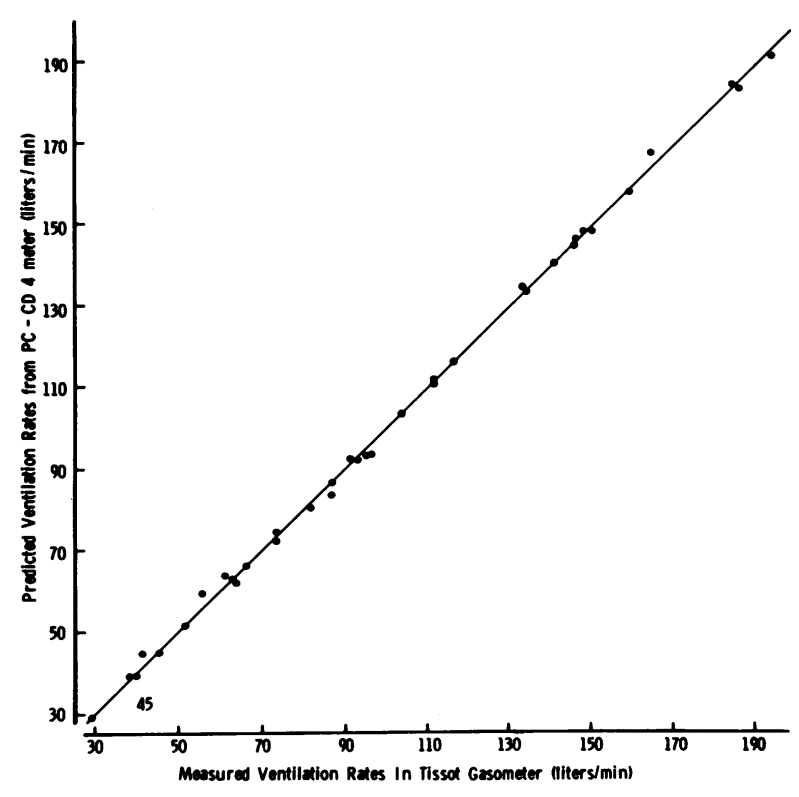

Fig. 2. Comparison of measured ventilation rates with predicted ventilation rates for the Tissot gasometer. Predicted rates were obtained by adjusting ventilatory rates from the Parkinson-Cowan CD-4 dry gas meter with a linear regression equation.

\section{Discussion}

Comparing the PC-CD4 with the Tissot values demonstrated that the data were related linearly over a $\dot{V}_{e}$ range of $30-193 \mathrm{I} / \mathrm{min}$. Both the regression equation and the small $\mathrm{SE}_{\mathrm{est}}$ found in this study correspond closely with the results published by Cunningham, Montoye and Welch (1969). It has been our experience, however, that each PC-CD4 meter must be calibrated independently and that they are not always linear over the range reported here. In such instances separate linear regression equations may be required for specific ranges of $\dot{V}_{e}$ or else non-linear regression equations may be used. It is also good practice to recalibrate the instrument at regular intervals.

It is apparent that the accuracy obtained with the PC-CD4, when corrected to Tissot values, is acceptable for most investigations. The ease of monitoring $\dot{V}_{e}$ continuously via a PC-CD4 meter in the system described by Mayhew (1973), which is also being used in our laboratory (Figure 3), facilitates gas collections to a one man operation.

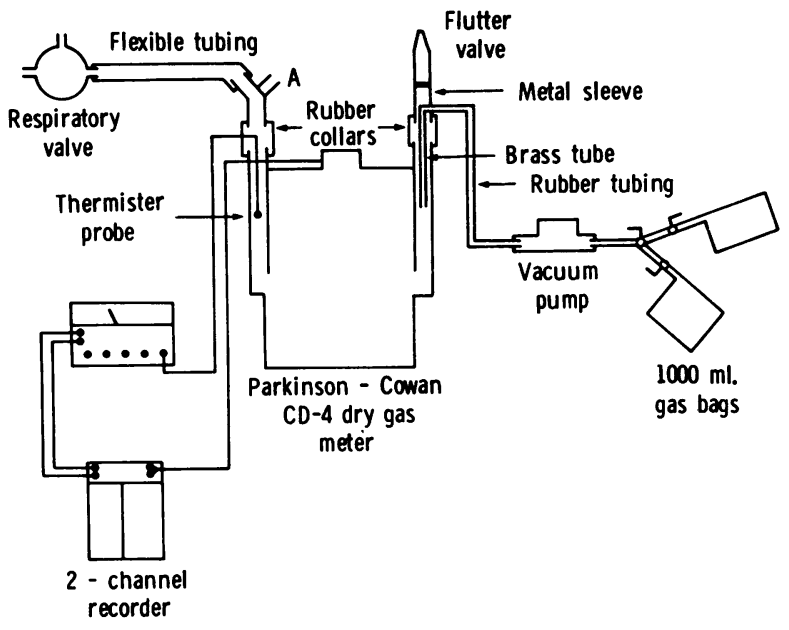

Fig. 3. Schematic representation of gas collection system.

\section{REFERENCES}

CUNNINGHAM, D. A., MONTOYE, H. J. and WELCH, H. G. "An evaluation of equipment for determining oxygen uptake." Res. Quart. 40: 851-856, 1969.

HENRY, F. M. and KATCH, V. L. "A dependable method of continuous recording of ventilation meter output." Res. Quart. 43: 243-246, 1972.

JOHNSON, R. E., et al. "A versatile system for measuring oxygen consumption in man." J.Appl.Physiol. 22: 377-379. 1967.

MAYHEW, J. L. "A reliable versatile system for assessing oxygen intake." Brit.J. Sports Med. 7: 360-362, 1973.

WILMORE, J. H. "A simplified method for obtaining aliquots of respiratory air." Res.Quart. 39: 824-828, 1968.

WILMORE, J. H. and COSTILL, D. L. "Semiautomated systems approach to the assessment of oxygen uptake during exercise." J.Appl.Physiol. 36: 618-620, 1974. 